\title{
On Inequality Applicable to Partial Dynamic Equations
}

\author{
Deepak B. Pachpatte \\ Department of Mathematics, Dr. B.A.M. University, Aurangabad, Maharashtra 431004, India
}

Correspondence should be addressed to Deepak B. Pachpatte; pachpatte@gmail.com

Received 12 February 2014; Accepted 26 March 2014; Published 15 April 2014

Academic Editor: Peiguang Wang

Copyright (C) 2014 Deepak B. Pachpatte. This is an open access article distributed under the Creative Commons Attribution License, which permits unrestricted use, distribution, and reproduction in any medium, provided the original work is properly cited.

The main objective of the paper is to study new integral inequality on time scales which is used for the study of some partial dynamic equations. Some applications of our results are also given.

\section{Introduction}

During past few decades many authors have established various dynamic inequalities useful in the development of differential and integral equations. Mathematical inequalities on time scales play an important role in the theory of dynamic equations. The study of time scale was initiated by Hilger [1] in 1990 in his Ph.D. thesis which unifies continuous and discrete calculus. Since then, many authors have studied various properties of dynamic equations on time scales [29].

In what follows, let $\mathbb{R}$ denotes the set of real numbers and let $\mathbb{T}$ denote the arbitrary time scales. Let $\mathbb{R}_{+}=[0, \infty)$, $\mathbb{T}_{1}=[0, a]$, and $\mathbb{T}_{2}=[0, b]$ be subsets of $\mathbb{R}$ and $\Omega=\mathbb{T}_{1} \times \mathbb{T}_{2}$. Let $C_{\mathrm{rd}}$ denote the set of rd-continuous function. The partial delta derivative of $v(x, y)$ for $(x, y) \in \Omega$ with respect to $x, y$, and $x y$ is denoted by $v^{\Delta_{1}}(x, y), v^{\Delta_{2}}(x, y)$, and $v^{\Delta_{1} \Delta_{2}}(x, y)=$ $v^{\Delta_{2} \Delta_{1}}(x, y)$. We assume here understanding of time scales calculus and notations. Further information about time scales calculus can be found in $[1,5,10]$.

We require the following lemmas given in $[5,6]$.

Lemma 1 (see [5], Theorem 2.6). Let $u \in C_{\mathrm{rd}}\left(\mathbb{T}, \mathbb{R}_{+}\right), a \in \mathbb{R}_{+}$, and

$$
u^{\Delta}(t) \leq a(t) u(t)
$$

for all $t \in \mathbb{T}^{k}$; then

$$
u(t) \leq u\left(t_{0}\right) e_{a}\left(t, t_{0}\right)
$$

for all $t \in \mathbb{T}^{k}$.
Lemma 2 (see [6], Lemma 2.1). Let $u, a, b \in C_{\mathrm{rd}}\left(\Omega, \mathbb{R}_{+}\right)$and $a(x, y)$ is nondecreasing in $(x, y) \in \Omega$ and

$$
u(x, y) \leq a(x, y)+\int_{x_{0}}^{x} \int_{y_{0}}^{y} b(s, t) u(s, t) \Delta t \Delta s
$$

for $(x, y) \in \Omega$; then

$$
u(x, y) \leq a(x, y) e_{\mathrm{Q}(x, y)}\left(x, x_{0}\right),
$$

where

$$
Q(x, y)=\int_{y_{0}}^{y} b(x, t) \Delta t
$$

for $(x, y) \in \Omega$.

\section{Main Results}

Now in this section we give our main results.

Theorem 3. Let $u(x, y), w(x, y), p(x, y), q(x, y), r(x, y) \in$ $C_{\mathrm{rd}}\left(\Omega, \mathbb{R}_{+}\right)$and suppose that

$$
\begin{aligned}
u(x, y) \leq c & +\int_{x_{0}}^{x} w(s, y) u(s, y) \Delta s+\int_{s_{0}}^{s} \int_{y_{0}}^{y} p(s, t) \\
\times & {\left[u(s, t)+\int_{s_{0}}^{s} \int_{t_{0}}^{t} q(\xi, \tau) u(\xi, \tau) \Delta \tau \Delta \xi\right.} \\
& \left.+\int_{a_{0}}^{a} \int_{b_{0}}^{b} r(\xi, \tau) u(\xi, \tau) \Delta \tau \Delta \xi\right] \Delta t \Delta s
\end{aligned}
$$


for $(x, y) \in \Omega$, where $c \geq 0$ is a constant. If

$$
g=\int_{a_{0}}^{a} \int_{b_{0}}^{b} r(\xi, \tau) A(\xi, \tau) e_{H(x, y)}\left(\xi, \tau_{0}\right) \Delta \tau \Delta \xi,
$$

where

$$
\begin{gathered}
H(x, y)=\int_{\tau_{0}}^{\tau} A(x, t)[p(x, t)+q(x, t)] \Delta t, \\
A(x, y)=e_{w(x, y)}\left(x, x_{0}\right),
\end{gathered}
$$

for $(x, y) \in \Omega$, then

$$
u(x, y) \leq \frac{c}{1-g} A(x, y) e_{H(x, y)}\left(x_{0}, x\right),
$$

for $(x, y) \in \Omega$.

Proof. Define a function $z(x, y)$ by

$$
\begin{aligned}
z(x, y)= & +\int_{x_{0}}^{x} \int_{y_{0}}^{y} p(s, t) \\
& \times\left[u(s, t) \times \int_{s_{0}}^{s} \int_{t_{0}}^{t} q(\xi, \tau) u(\xi, \tau) \Delta \tau \Delta \xi\right. \\
& \left.\quad+\int_{s_{0}}^{s} \int_{t_{0}}^{t} r(\xi, \tau) u(\xi, \tau) \Delta \tau \Delta \xi\right] \Delta t \Delta s .
\end{aligned}
$$

Then (6) is

$$
u(x, y) \leq z(x, y)+\int_{x_{0}}^{x} w(s, y) u(s, y) \Delta s
$$

It is easy to see that $z(x, y)$ is nonnegative, rd-continuous, and nondecreasing function for $(x, y) \in \Omega$. Treating $y$ fixed and using Lemma 1 we get

$$
u(x, y) \leq A(x, y) z(x, y)
$$

for $(x, y) \in \Omega$, where $A(x, y)$ is defined by (9). From (11), (12), and the fact that $A(x, y) \geq 1$, we have

$$
\begin{array}{rl}
z(x, y) \leq c+\int_{x_{0}}^{x} \int_{y_{0}}^{y} p(s, t) & \\
\times & {[A(s, t) z(s, t)} \\
& +\int_{s_{0}}^{s} \int_{t_{0}}^{t} q(\xi, \tau) A(\xi, \tau) \\
\times & \times(\xi, \tau) \Delta \tau \Delta \xi \\
& +\int_{a_{0}}^{a} \int_{b_{0}}^{b} r(\xi, \tau) A(\xi, \tau) \\
\leq c+\int_{x_{0}}^{x} \int_{y_{0}}^{y} & p(s, t) A(s, t) \\
\times & {\left[z(s, t)+\int_{s_{0}}^{s} \int_{t_{0}}^{t} q(\xi, \tau) A(\xi, \tau) \Delta \tau \Delta \xi\right] \Delta t \Delta s}
\end{array}
$$

$$
\begin{aligned}
+\int_{a_{0}}^{a} \int_{b_{0}}^{b} h(\xi, \tau) A(\xi, \tau) \\
\quad \times z(\xi, \tau) \Delta \tau \Delta \xi] \Delta t \Delta s
\end{aligned}
$$

Define a function $v(x, y)$ by right hand side of (14). Then $v(0, y)=v(x, 0)=c, z(x, y) \leq v(x, y)$. One has

$$
\begin{aligned}
v^{\Delta_{2} \Delta_{1}}= & p(x, y) A(x, y) \\
\times & \quad\left[z(x, y)+\int_{x_{0}}^{x} \int_{y_{0}}^{y} q(\xi, \tau) A(\xi, \tau) z(\xi, \tau) \Delta \tau \Delta \xi\right. \\
& \left.\quad+\int_{a_{0}}^{a} \int_{b_{0}}^{b} r(\xi, \tau) A(\xi, \tau) z(\xi, \tau) \Delta \tau \Delta \xi\right] \\
\leq & p(x, y) A(x, y) \\
& \times\left[v(x, y)+\int_{x_{0}}^{x} \int_{y_{0}}^{y} q(\xi, \tau) A(\xi, \tau) v(\xi, \tau) \Delta \tau \Delta \xi\right. \\
& \left.\quad+\int_{a_{0}}^{a} \int_{b_{0}}^{b} r(\xi, \tau) A(\xi, \tau) v(\xi, \tau) \Delta \tau \Delta \xi\right] .
\end{aligned}
$$

Define a function $f(x, y)$ by

$$
\begin{aligned}
f(x, y)= & v(x, y)+\int_{x_{0}}^{x} \int_{y_{0}}^{y} q(\xi, \tau) A(\xi, \tau) v(\xi, \tau) \Delta \tau \Delta \xi \\
& +\int_{a_{0}}^{a} \int_{b_{0}}^{b} r(\xi, \tau) A(\xi, \tau) v(\xi, \tau) \Delta \tau \Delta \xi
\end{aligned}
$$

then $v(x, y) \leq f(x, y), v^{\Delta_{2} \Delta_{1}}(x, y) \leq p(x, y) A(x, y) f(x, y)$,

$$
\begin{aligned}
f\left(x_{0}, y\right)= & f\left(x, y_{0}\right) \\
= & c+\int_{a_{0}}^{a} \int_{b_{0}}^{b} r(\xi, \tau) A(\xi, \tau) v(\xi, \tau) \Delta \tau \Delta \xi \\
= & M(\text { say }), \\
f^{\Delta_{2} \Delta_{1}}(x, y)= & v^{\Delta_{2} \Delta_{1}}(x, y) \\
& +q(x, y) A(x, y) v(x, y) \\
\leq & p(x, y) A(x, y) f(x, y) \\
& +q(x, y) A(x, y) f(x, y) \\
= & A(x, y)[p(x, y)+q(x, y)] f(x, y) .
\end{aligned}
$$

By keeping $\mathbf{x}$ fixed in (18), taking $y=t$ and delta integrating with second variable from $y_{0}$ to $y$. Using the fact that $f^{\Delta_{1}}\left(x, y_{0}\right)=0$ and $f(x, y)$ is nondecreasing in $(x, y) \in \Omega$, we have

$$
\begin{aligned}
f^{\Delta_{1}}(x, y) & \leq \int_{y_{0}}^{y} A(x, t)[p(x, t)+q(x, t)] f(x, t) \Delta t \\
& \leq f(x, y) \int_{y_{0}}^{y} A(x, t)[p(x, t)+q(x, t)] \Delta t
\end{aligned}
$$

Let

$$
\bar{Q}(x, y)=\int_{y_{0}}^{y} A(x, t)[p(x, t)+q(x, t)] \Delta t
$$


then (20) gives

$$
f^{\Delta_{1}}(x, y) \leq f(x, y) \bar{Q}(x, y) .
$$

Now treating $y$ fixed in (21) and applying Lemma 1, we have

$$
f(x, y) \leq M e_{\bar{Q}(x, y)}\left(x, x_{0}\right) .
$$

From (18), (22), and (7), it is easy to see that

$$
M \leq \frac{c}{1-g} .
$$

Using (23) in (22) and the fact that $z(x, y) \leq v(x, y)$ and $z(x, y) \leq A(x, y) v(x, y)$ we get the inequality in (10).

This completes the proof.

\section{Applications}

Now we give some application of theorem to study properties of solutions of initial value problem:

$$
\begin{gathered}
u^{\Delta_{2} \Delta_{1}}(x, y)=(w(x, y) u(x, y))^{\Delta_{2}} \\
+G(x, y, u(x, y), \\
\left.\int_{a_{0}}^{a} \int_{b_{0}}^{b} h(x, y, \xi, \tau, u(\xi, \tau)) \Delta \tau \Delta \xi\right), \\
u\left(x, y_{0}\right)=\alpha(x), \\
u\left(x_{0}, y\right)=\beta(y), \\
\alpha\left(x_{0}\right)=\beta\left(y_{0}\right)=0,
\end{gathered}
$$

where $\alpha \in C_{\text {rd }}\left(\mathbb{T}_{1}, \mathbb{R}\right), \beta \in C_{\text {rd }}\left(\mathbb{T}_{2}, \mathbb{R}\right)$ for $0 \leq \xi \leq x, 0 \leq \tau \leq$ $y, h \in C_{\mathrm{rd}}\left(\Omega^{2} \times \mathbb{R}, \mathbb{R}\right), G \in C_{\mathrm{rd}}\left(\Omega \times \mathbb{R}^{2}, \mathbb{R}\right), p \in C_{\mathrm{rd}}(\Omega, \mathbb{R})$ is delta differentiable with respect to $y$.

We observe that (24) is equivalent to

$$
\begin{aligned}
& u(x, y) \\
& =F(x, y)+\int_{x_{0}}^{x} w(s, y) u(s, y) \Delta s \\
& \quad+\int_{x_{0}}^{x} \int_{y_{0}}^{y} G(s, t, u(s, t), \\
& \left.\quad \int_{a_{0}}^{a} \int_{b_{0}}^{b} h(s, t, \xi, \tau, u(\xi, \tau)) \Delta \tau \Delta \xi\right) \Delta t \Delta s,
\end{aligned}
$$

where

$$
F(x, y)=\alpha(x)+\beta(y)-\int_{x_{0}}^{x} p\left(s, y_{0}\right) \alpha(s) \Delta s .
$$

The following theorem deals with estimate on solution (24).

\section{Theorem 4. Suppose}

$$
\begin{gathered}
|F(x, y)| \leq c, \\
|h(x, y, s, t, u)| \leq k(x, y) r(s, t)|u|, \\
|G(x, y, u, \bar{u})| \leq p(x, y)(|u|+|\bar{u}|),
\end{gathered}
$$

where $p, r, c$ which are as in Theorem 3 and $k(x, y)$ is $r d$ continuous function defined on $\Omega$ such that $k(x, y) \geq 1$. Let

$$
g_{0}=\int_{a_{0}}^{a} \int_{b_{0}}^{b} r(\xi, \tau) A(\xi, \tau) e_{\bar{H}(x, y)}\left(\xi, \tau_{0}\right) \Delta \tau \Delta \xi,
$$

where

$$
\begin{gathered}
\bar{H}(x, y)=\int_{t_{0}}^{\tau} A(x, t) p(x, t) k(x, t) \Delta t, \\
\bar{A}(x, y)=e_{w(r, y)}\left(x, x_{0}\right),
\end{gathered}
$$

for $(x, y) \in \Omega$. If $u(x, y)$ is any solution of $(24)$, then

$$
u(x, y) \leq \frac{c}{1-g_{0}} \bar{A}(x, y) e_{\bar{H}(x, y)}\left(x, x_{0}\right),
$$

where $(x, y) \in \Omega$.

Proof. The solution $u(x, y)$ of (24) satisfies (25). Using (27) in (25) we have

$$
\begin{aligned}
& |u(x, y)| \\
& \leq c+\int_{x_{0}}^{x}|w(s, y)||u(s, y)| \Delta s \\
& +\int_{x_{0}}^{x} \int_{y_{0}}^{y} p(s, t) \\
& \times[|u(s, t)| \\
& +\int_{a_{0}}^{a} \int_{b_{0}}^{b} k(s, t) r(\xi, \tau) \\
& \times|u(\xi, \tau)| \Delta \tau \Delta \xi] \Delta t \Delta s \\
& \leq c+\int_{x_{0}}^{x}|w(s, y)||u(s, y)| \Delta s \\
& +\int_{x_{0}}^{x} \int_{y_{0}}^{y} p(s, t) k(s, t) \\
& \times[|u(s, t)| \\
& +\int_{a_{0}}^{a} \int_{b_{0}}^{b} r(\xi, \tau) \\
& \times|u(\xi, \tau)| \Delta \tau \Delta \xi] \Delta t \Delta s .
\end{aligned}
$$

Now an application of Theorem 3 (with $g=0$ ) to (32) yields (30).

This completes the proof.

Now we establish the uniqueness of solutions of (24).

Theorem 5. Suppose that

$$
\begin{array}{r}
|h(x, y, s, t, u)-h(x, y, s, t, \bar{u})| \leq k(x, y) r(s, t)|u-\bar{u}|, \\
|G(x, y, u, \bar{u})-G(x, y, v, \bar{v})| \leq p(x, y)(|u-v|+|\bar{u}-\bar{v}|),
\end{array}
$$

where $k, p$, and $r$ are as in Theorem 4. Let $g_{0}$ and $\bar{A}(x, y)$ be as in (28) and (30). Then (24) has at most one solution on G. 
Proof. Let $u(x, y)$ and $v(x, y)$ be two solutions of (24) on $\Omega$; then we have

$$
\begin{aligned}
& u(x, y)-v(x, y) \\
& =\int_{x_{0}}^{x} w(x, y)\{u(s, y)-v(s, y)\} \Delta s \\
& \quad+\int_{x_{0}}^{x} \int_{y_{0}}^{y}\{G(s, t, u(s, t), \\
& \left.\quad \int_{a_{0}}^{a} \int_{b_{0}}^{b} h(s, t, \xi, \tau, u(\xi, \tau)) \Delta \tau \Delta \xi\right) \\
& \quad-G(s, t, v(s, t), \\
& \left.\left.\int_{a_{0}}^{a} \int_{b_{0}}^{b} h(s, t, \xi, \tau, v(\xi, \tau)) \Delta \tau \Delta \xi\right)\right\} \Delta t \Delta s .
\end{aligned}
$$

From (34) and (33) we obtain

$$
\begin{aligned}
& |u(x, y)-v(x, y)| \\
& \leq \int_{x_{0}}^{x}|w(s, y)||u(s, y)-v(s, y)| \Delta s \\
& +\int_{x_{0}}^{x} \int_{y_{0}}^{y} p(s, t) \\
& \times(|u(s, t)-v(s, t)|+k(s, t) \\
& \quad \times \int_{a_{0}}^{a} \int_{b_{0}}^{b} r(\xi, \tau) \\
& \times|u(\xi, \tau)-v(\xi, \tau)| \Delta \tau \Delta \xi) \Delta t \Delta s .
\end{aligned}
$$

Applying Theorem 3 (with $c=0, g=0$ ) yields

$$
|u(x, y)-v(x, y)| \leq 0 .
$$

Therefore $u(x, y) \leq v(x, y)$; there is at most one solution of (24) in $\Omega$.

\section{Conflict of Interests}

The author declares that there is no conflict of interests regarding the publication of this paper.

\section{References}

[1] S. Hilger, "Analysis on measure chains-a unified approach to continuous and discrete calculus," Results in Mathematics, vol. 18, no. 1-2, pp. 18-56, 1990.

[2] S. András and A. Mészáros, "Wendroff type inequalities on time scales via Picard operators," Mathematical Inequalities \& Applications, vol. 16, no. 1, pp. 159-174, 2013.

[3] D. R. Anderson, "Dynamic double integral inequalities in two independent variables on time scales," Journal of Mathematical Inequalities, vol. 2, no. 2, pp. 163-184, 2008.

[4] D. R. Anderson, "Nonlinear dynamic integral inequalities in two independent variables on time scale pairs," Advances in Dynamical Systems and Applications, vol. 3, no. 1, pp. 1-13, 2008.
[5] E. Akin-Bohner, M. Bohner, and F. Akin, "Pachpatte inequalities on time scales," Journal of Inequalities in Pure and Applied Mathematics, vol. 6, no. 1, article 6, 2005.

[6] R. A. C. Ferreira and D. F. M. Torres, "Some linear and nonlinear integral inequalities on time scales in two independent variables," Nonlinear Dynamics and Systems Theory, vol. 9, no. 2, pp. 161-169, 2009.

[7] D. B. Pachpatte, "Explicit estimates on integral inequalities with time scale," Journal of Inequalities in Pure and Applied Mathematics, vol. 7, no. 4, article 143, 2006.

[8] D. B. Pachpatte, "Properties of solutions to nonlinear dynamic integral equations on time scales," Electronic Journal of Differential Equations, vol. 2008, no. 136, pp. 1-8, 2008.

[9] D. B. Pachpatte, "Integral inequalitys for partial dynamic equations on time scales," Electronic Journal of Differential Equations, vol. 2012, no. 50, pp. 1-7, 2012.

[10] M. Bohner and A. Peterson, Dynamic Equations on Time Scales, Birkhäuser, Boston, Mass, USA, 2001. 


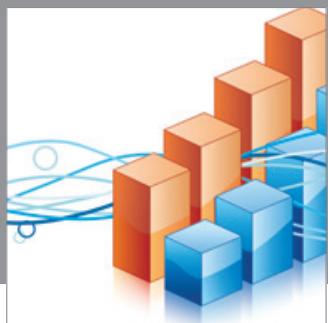

Advances in

Operations Research

mansans

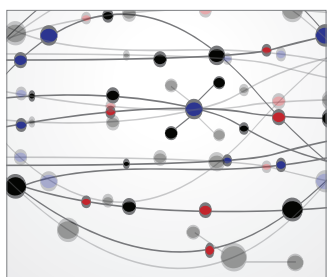

The Scientific World Journal
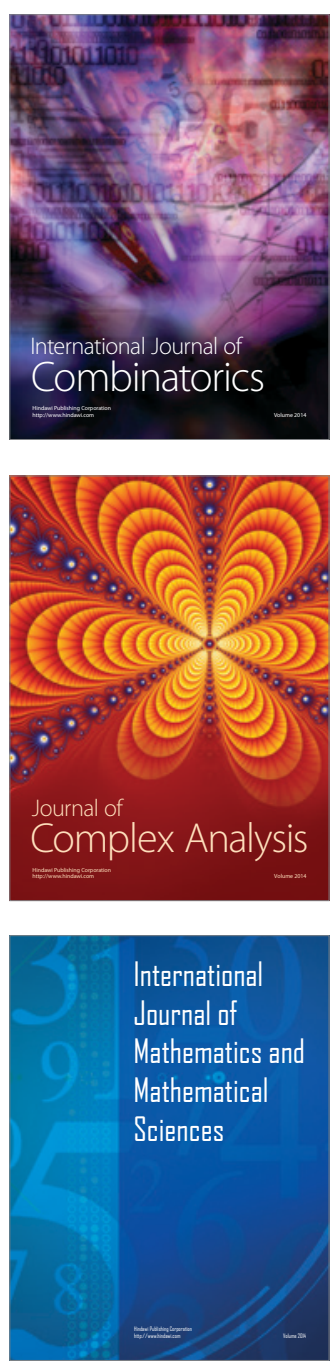
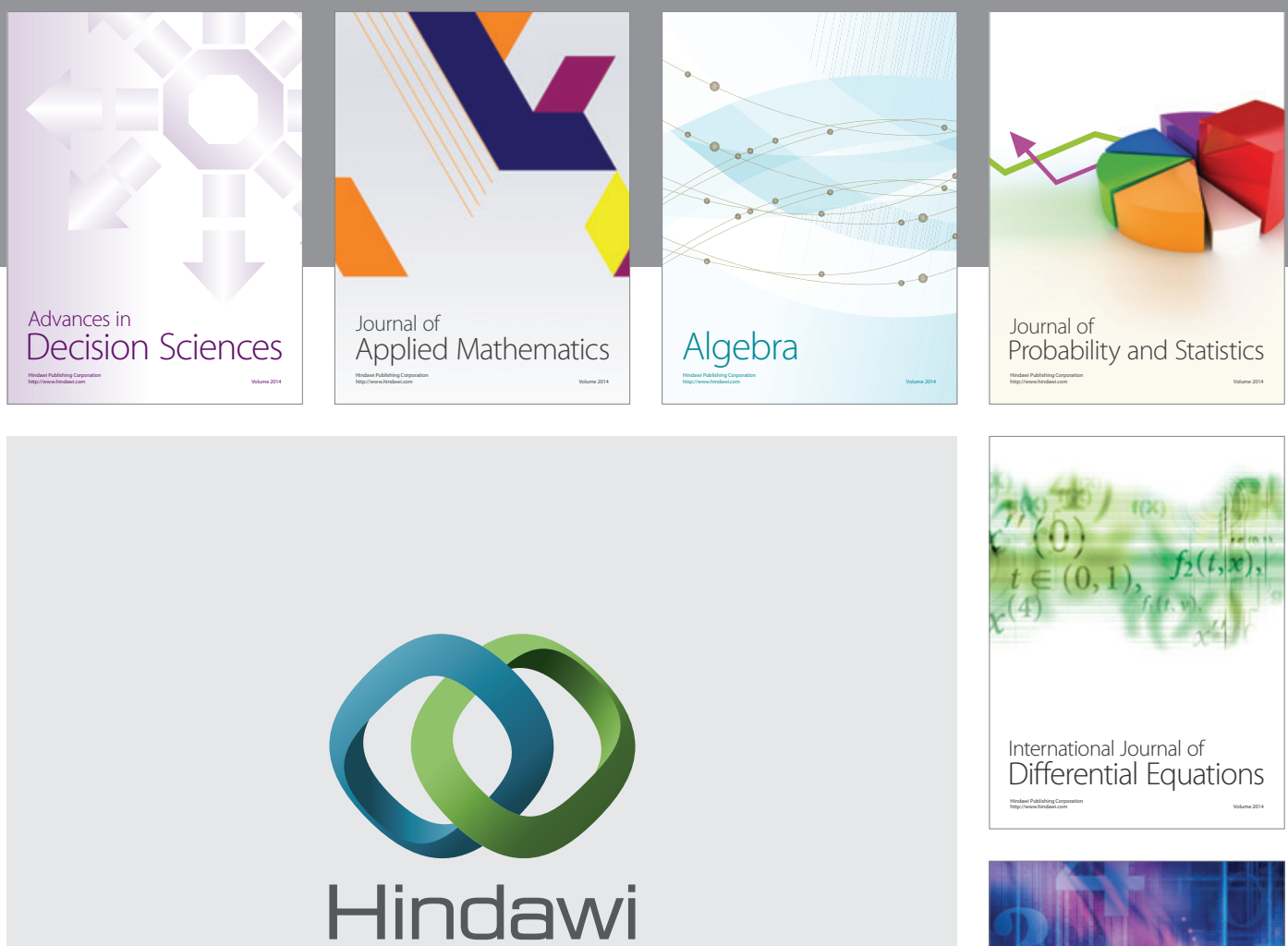

Submit your manuscripts at http://www.hindawi.com
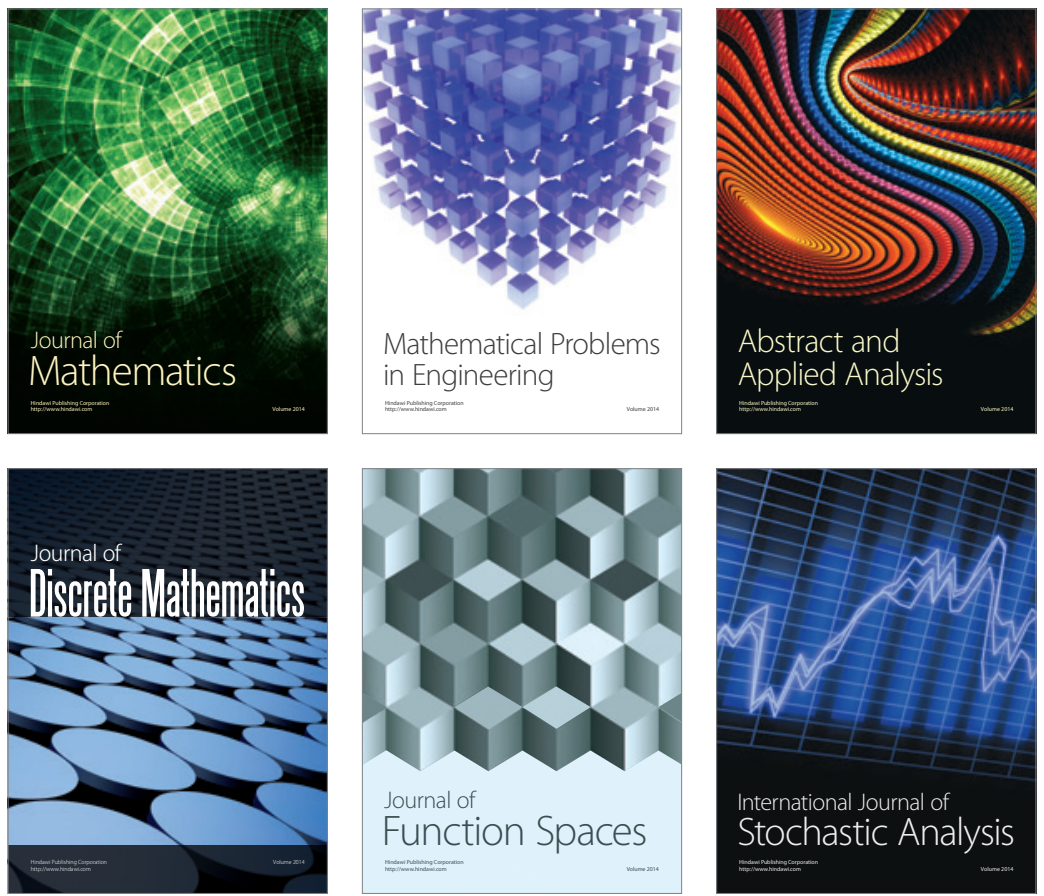

Journal of

Function Spaces

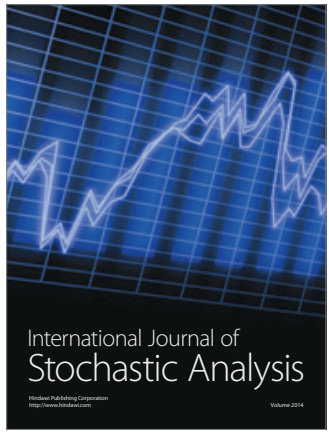

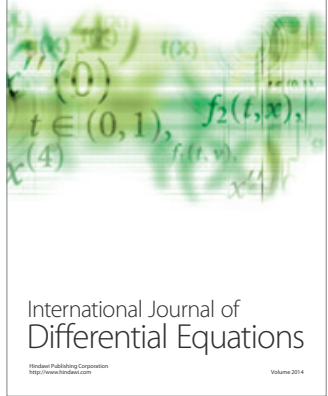
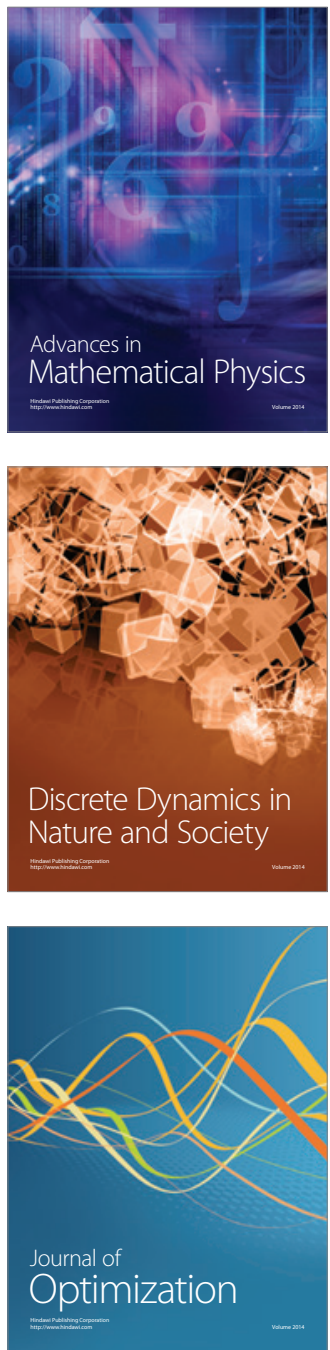